\title{
Ultrafast terahertz probes of transient conducting and insulating phases within an electron-hole gas
}

\author{
R. A. Kaindl*, M. A. Carnahan*, D. Hägele*t, R. Lövenich*, and D. S. Chemla* \\ * Department of Physics, University of California at Berkeley and Materials Sciences \\ Division, E. O. Lawrence Berkeley National Laboratory, Berkeley, CA 94720, USA \\ $\dagger$ Institut für Festkörperphysik, Universität Hannover, Appelstraße 2, 30167 Hannover, \\ Germany
}

Many-body systems in nature exhibit complexity and self-organization arising from seemingly simple laws. The long-range Coulomb interaction between electrical charges generates a plethora of bound states in matter, ranging from the hydrogen atom to complex biochemical structures. Semiconductors form an ideal laboratory for studying many-body interactions of quasi-particles among themselves and with lattice vibrations and light ${ }^{1-4}$. Oppositely charged electron and hole quasi-particles can coexist in an ionized but correlated plasma, or form bound hydrogen-like pairs called excitons which strongly affect physical properties ${ }^{5,6}$. The pathways between such states however remain elusive in near-visible optical experiments that detect a subset of excitons with vanishing center-of-mass momenta. In contrast, transitions between internal exciton levels which occur in the far-infrared at terahertz $\left(10^{12} s^{-1}\right)$ frequencies ${ }^{7-9}$ are independent of this restriction suggesting ${ }^{10}$ their use as a novel probe of pair dynamics. Here, we employ an ultrafast terahertz probe to directly investigate the dynamical interplay of optically-generated excitons and unbound electron-hole pairs in GaAs quantum wells. Our observations witness an unexpected quasi-instantaneous excitonic enhancement, reveal formation of insulating excitons on a hundred picosecond timescale and manifest conditions under which excitonic populations prevail. 
In semiconductors, optical excitation can promote electron (e) quasi-particles from the highest filled valence band into the empty conduction band separated by an electron-Volt $(\mathrm{eV})$ sized bandgap. The Coulomb interaction between these depleted electronic-states, called holes $(h)$, and the excited electrons can result in the formation of hydrogen-like bound $e-h$ states called excitons ${ }^{3}$. The binding energy of the lowest state is given by $\mathrm{E}_{b} \propto \frac{\mu}{m_{0} \varepsilon^{2}} R y$ where $\mu$ is the reduced pair effective mass, $\varepsilon$ the dielectric constant, $\mathrm{m}_{\mathrm{o}}$ the bare electron mass, and $1 \mathrm{Ry} \approx 13.6 \mathrm{eV}$ the hydrogen atom Rydberg energy. Small reduced masses and large dielectric constants of semiconductors ( $\mu \approx 5 \% \mathrm{~m}_{0}$ and $\varepsilon \approx 13$ in prototypical GaAs) result in binding energies of barely a few $\mathrm{meV}$, orders of magnitude below the valence-to-conduction band transition. Hence, the natural scale for electromagnetic transitions between internal exciton levels is the farinfrared, terahertz $(\mathrm{THz})$ spectral range, where $1 \mathrm{THz}=10^{12} \mathrm{~Hz}$ corresponds to a photon energy $\approx 4.1 \mathrm{meV}$.

Photoexcitation of even low densities of quasiparticles in condensed matter results in complex many-body states which arise from the interactions among quasiparticles and with other elementary quanta of vibrations (phonons) or light (photons). In the past, the dynamics of excited semiconductors was often investigated by absorption, photoluminescence, or nonlinear wave mixing experiments close to the bandgap ${ }^{1,2}$. These techniques are based on creation and/or destruction of excitons and - because of the photon's small momentum-probe $e$ - $h$ states with negligible center-of-mass momentum K (Fig 1a). Absorption in that spectral range measures the generation of $e-h$ pairs and is consequently only indirectly influenced by carrier and excitonic populations. In contrast, $\mathrm{THz}$ transitions can measure populations of excitons or conducting charges already present in the sample - photogenerated or introduced by doping - and at center-of-mass momenta well outside the optically accessible range. ${ }^{10}$ This opens new opportunities for precise spectroscopy of low-energy excitation 
dynamics. Recently, the buildup of Coulomb screening in an electron-hole plasma was reported $^{11}$. Furthermore, because time-resolved $\mathrm{THz}$ experiments measure both the real and the imaginary parts of the dielectric function they impose strict conditions on theoretical models ${ }^{11,12}$. These arguments strongly motivate using internal exciton transitions to probe exciton dynamics. Early studies observed internal exciton transitions $^{7-9}$ but the dynamics remains largely unexplored. Here, we carry out such experiments where changes in a temporally pulsed $\mathrm{THz}$ field are detected after it has traversed an optically-excited semiconductor (Fig 1b). Time-resolved studies under various conditions reveal new and unexpected aspects of such fundamental dynamical processes as formation, population relaxation, and ionization of excitons.

In our experiments, the photexcited quasi-particles are quantum confined within semiconductor quantum well nano-layers. We investigate a stack of ten high-quality, undoped $14-\mathrm{nm}$ wide GaAs wells separated by 10 -nm wide $\mathrm{Al}_{0.3} \mathrm{Ga}_{0.7} \mathrm{As}$ barriers ${ }^{13}$. The low-temperature near-infrared absorption spectrum (Fig. 2a) is dominated by the absorption line of 1 s excitons comprised of electrons and heavy-holes (HH). With increasing photon energy, other exciton lines appear followed by the inter-band $e-h$ pair continuum. The sample is excited with ultrashort near-infrared pump pulses from a 250-kHz Ti:sapphire amplifier spectrally narrowed to $2 \mathrm{meV}$ bandwidth. Picosecond $\mathrm{THz}$ probe pulses are generated from the same source by optical rectification and detected via electro-optic sampling in ZnTe. For each fixed pump-probe time delay $\Delta t$ between the arrival of pump and probe pulses on the sample, Fig 1b, we probe the $\mathrm{THz}$ field $E(t)$ transmitted through the unexcited sample and the pump-induced field change $\Delta E(t)$. From that, the change $\Delta \tilde{\sigma}(\omega)$ of the frequency-dependent conductivity in the quantum wells is obtained by Fourier transform of the fields and straightforward electrodynamical relations. Extending earlier single-layer treatments ${ }^{14}$, we account for Fresnel phase-shifts at long wavelengths relevant for our multilayer structure. The complex optical conductivity, expressed as 


$$
\tilde{\sigma}(\omega)=\sigma_{1}(\omega)+i \frac{\omega}{4 \pi}\left[1-\varepsilon_{1}(\omega)\right]
$$

determines the current response $\mathrm{J}(\omega)=\tilde{\sigma}(\omega) \mathrm{E}(\omega)$ of the many-body system to the incident transverse electromagnetic field $\mathrm{E}(\omega)$. Its real part $\sigma_{1}(\omega)$ is a measure of the absorbed power density, and the imaginary part is given in terms of the real dielectric function $\varepsilon_{1}(\omega)$. Through Kramers-Kronig relations ${ }^{15}$ changes in $\varepsilon_{1}(\omega)$ are fundamentally linked to a corresponding low-frequency limit, induced conductivity via

$$
\Delta \sigma_{1}(\omega<1 / \tau) \approx-\frac{1}{2 \pi^{2}} \int_{0}^{\infty} \Delta \varepsilon_{1}\left(\omega^{\prime}\right) d \omega^{\prime},
$$

where $1 / \tau$ is the transport scattering rate. Thus, a nonvanishing integral due to an imbalance in $\Delta \varepsilon_{1}$ signifies a conducting phase. The availability of both parts of $\tilde{\sigma}(\omega)$, on equal footing, is key to understanding the physical state.

Resonant excitation of a density of $10^{10} \mathrm{~cm}^{-2} \mathrm{HH}$ excitons at low lattice temperatures $T_{L}=6 \mathrm{~K}$ leads to the appearance of a strong asymmetric peak in $\Delta \sigma_{1}(\omega)$ around $\hbar \omega \approx 7 \mathrm{meV}$, as shown in Fig. $2 \mathrm{~b}$ (dots) $5 \mathrm{ps}$ after excitation. Likewise the corresponding $\Delta \varepsilon_{1}(\omega)$, Fig. 2c, indicates the formation of this new low-energy oscillator. This can be explained as follows: initially, the pump creates coherent polarization waves which experience scattering with phonons and defects and within a few ps transform into an incoherent low-energy $1 \mathrm{~s}$ exciton population (for the near-gap coherent response, see Ref. 13). The THz field then probes the internal degrees of freedom of these excitons. Calculations taking into account the quasi-two dimensional exciton wavefunctions ${ }^{16}$ agree closely with this shape (black lines, Fig 2b,c). Specifically, the peak around $7 \mathrm{meV}$ is attributed to transition from the $1 \mathrm{~s} \rightarrow 2 \mathrm{p}$ level. Its energy correlates well with reported binding energies ${ }^{17}$. The broad shoulder corresponds to transitions into higher-energy bound and continuum states. The insulating nature of the charge-neutral excitons is apparent from the vanishing lowenergy conductivity. A completely different behavior is seen after excitation of unbound $e-h$ pairs in the continuum (Figs $2 \mathrm{~d}, \mathrm{e}$ ). Here, a high lattice temperature $\mathrm{T}=300 \mathrm{~K}$ is 
chosen to ensure minimal survival of excitonic correlations and indeed the response is well-described by the Drude model of dissipative charge motion (black lines). It is important to note that unlike doped semiconductors, the observation of this nonvanishing Drude-like response does not rely on impurities for momentum conservation. Rather, electron-hole collisions provide efficient scattering channels due to the large mass difference between these quasi-particles. ${ }^{18}$ The conducting nature of this state is evident from both the non-vanishing low-energy conductivity and the all-negative dielectric function change.

The dynamics after resonant excitation depends strongly on lattice temperature as shown in Fig. 3a. At low temperature $\left(\mathrm{T}_{\mathrm{L}}=6 \mathrm{~K}\right)$ the conductivity reduces in amplitude but retains its peaked lineshape. Hence its decay follows the recombination of exciton populations within several hundred picoseconds. At higher temperatures $\left(\mathrm{T}_{\mathrm{L}}=30 \mathrm{~K}\right.$ and $60 \mathrm{~K})$ the dynamics changes considerably. A fast decay of the peak into a broad response is observed, with a transformation rate that increases strongly with $T_{L}$. Figure $3 \mathrm{~b}$ shows the induced $\Delta \sigma_{1}(\omega)$ and $\Delta \varepsilon_{1}(\omega)$ spectra for $\mathrm{T}_{\mathrm{L}}=60 \mathrm{~K}$. Directly after excitation $(\Delta t=1 \mathrm{ps})$ a distinct $\mathrm{THz}$ exciton line is observed in $\Delta \sigma_{1}(\omega)$, but with increasing $\Delta \mathrm{t}$ it broadens considerably and eventually decays into the broad response. Correspondingly, the $\Delta \varepsilon_{1}(\omega)$ profile evolves from the characteristic oscillator dispersion into a negative Drude-like spectrum. Thus the dynamics is governed by ionization ${ }^{19}$ of the cold excitons, via absorption of thermal acoustic and optical phonons. The $\mathrm{THz}$ conductivity response of the ionized state $(\Delta t=100 \mathrm{ps}$, Fig. 3b), however, is not sufficiently described by a Drude model (red line): above $6 \mathrm{meV}$ it exhibits a strong enhancement at the photoionization threshold which indicates the survival of excitonic correlations.

Non-resonant excitation above the bandgap into the unbound $e$ - $h$ continuum leads to a qualitatively different behavior (Fig. 4a). A broadband $\mathrm{THz}$ response is observed at 
early time delays. The strongly negative $\Delta \varepsilon_{1}(\omega)$ and large low-frequency conductivity signify a predominantly conducting $e-h$ gas. Surprisingly however, a strong excitonic enhancement again exists immediately after excitation $(\Delta t=0 \mathrm{ps})$ as evident from the excess conductivity in $\Delta \sigma_{1}(\omega)$ around the exciton photoionization threshold. The spectra resemble the time-reversed ionization dynamics of Fig. 3b, although with a completely different evolution rate. Features around $7 \mathrm{meV}$ build up on a timescale of hundreds of ps, during which characteristic sharp exciton line shapes emerge and Drude-like contributions abate. Around $\Delta \mathrm{t}=1000 \mathrm{ps}$, the exciton response is fully restored.

A particularly intriguing aspect concerns the observation of two very distinct timescales in exciton formation, associated with the quasi-instantaneous appearance of a strong excitonic enhancement, and the much slower full transformation from the photoexcited conducting $e-h$ gas to a charge-neutral excitonic phase. Previous theories of exciton formation consider only the extremely dilute limit of independent $e$ - $h$ pairs interacting with phonons ${ }^{20-22}$. This simplified picture cannot account for the complex many-body dynamics observed in real systems and measured in our experiments, Fig 4. Because excitons are composed of the same states as unbound - yet still interacting quasi-particles, they cannot be treated as separate species. Within a photoexcited $e-h$ gas ultrafast Coulomb interactions introduce fundamentally new properties through two-pair and higher correlations. Some of these can be intuitively visualized by processes such as an unbound $e-h$ pair forming an excitonic state after transferring energy and momentum to other carriers (and its reversed process), or redistribution among bound $e$ - $h$ pairs due to collisions with another bound or unbound $e-h$ pair. Such Coulomb interactions naturally explain the observed quasi-instantaneous emergence of an excitonic enhancement around $\Delta \mathrm{t}=0 \mathrm{ps}$ in the optical conductivity and its evolution. Our spectra - including intermediate delay times - do not exhibit a sharp exciton structure growing on a broad Drude background, but reveal instead a conglomerate response with timedependent broadening. This is not explained by center-of-mass dispersion or phonon 
scattering that contribute less than $\approx 1 \mathrm{meV}$ to broadening ${ }^{23,24}$ but rather reflects the transient nature of the correlations as mediated by Coulomb interactions.

Interactions within the electronic system can, however, only transiently redistribute its internal energy. A full transformation of unbound e-h pairs into excitons demands a phonon bath in which to permanently release the binding energy and momentum. Photoluminescence measures exciton momentum relaxation to $\mathrm{K} \approx 0$ rather than their formation, and unbound pairs are hard to detect ${ }^{25-28}$. In our experiment, a natural distinction between unbound and excitonic many-particle states arises from the low-energy response that yields a collective measure of charge conductance. The decay of conducting properties observed here is a direct indicator of the increasingly correlated motion of oppositely charged quasi-particles. This clear distinction arises from the induced dielectric function $\Delta \varepsilon_{1}(\omega)$, which even for a broadened oscillator is always positive at frequencies below its resonance, but is generally negative for a Drude-like conductor. Figure $4 \mathrm{~b}$ illustrates the dynamics of $\Delta \varepsilon_{1}$ at $1 \mathrm{THz}(\hbar \omega \approx 4 \mathrm{meV})$. The slow monotonic increase from initially negative to positive values is linked to the slowly decreasing contribution from the conducting $e-h$ gas. This gradual process unambigously shows that exciton formation is characterized by the coexistence of excitons and $e$ - $h$ pairs over several 100 ps.

Binding into excitons eventually forms an insulating quantum state with a fundamental excitation gap equal to the exciton binding energy. In this way, the $\mathrm{THz}$ dynamics in Fig. 4 provides evidence for a metal-to-insulator transition ${ }^{29,30}$ which arises here from the decay of a transient conductive state. In the GaAs wells studied here, the fragile nature of the insulating electronic ground state is evidenced in the stark contrast between its nanosecond persistence after resonant excitation at low temperatures, and the quick, picosecond ionization observed at elevated lattice temperatures. We envision 
that the $\mathrm{THz}$ response uncovered here will enable studies of new non-equilibrium states that remain elusive within near-infrared optics.

1. Shah, J. Ultrafast Spectroscopy of Semiconductors and Semiconductor Nanostructures. (Springer Verlag, 1999).

2. Chemla D. S. \& Shah. J. Many-body and correlation effects in semiconductors. Nature 411, 549-557 (2001).

3. Rashba, E. I. Excitons. (North-Holland Publ. Co., Amsterdam, 1982).

4. Perakis, I. Exciton developments. Nature 417, 33 (2002).

5. Lundstrom, T., Schoenfeld, W., Lee, H. \& Petroff, P.M. Exciton storage in semiconductor self-assembled quantum dots. Science 286, 2312-2314 (1999).

6. Bayer, M., Stern, O., Hawrylak, P., Fafard, S. \& Forchel, A. Hidden symmetries in the energy levels of excitonic 'artificial atoms'. Nature 405, 923-926 (2000).

7. Groeneveld, R. H. M. \& Grischkowsky, D. Picosecond time-resolved far-infrared experiments on carriers and excitons in GaAs-AlGaAs multiple quantum wells. J. Opt. Soc. Am. B11, 2502-2507 (1994).

8. Timusk, T. Far-infrared absorption study of exciton ionization in germanium. Phys. Rev. B13, 3511-3514 (1976).

9. Cerne, J. et al. Terahertz Dynamics of Excitons in GaAs/AlGaAs Quantum Wells. Phys. Rev. Lett. 77, 1131-1134 (1996).

10. Kira, M., Hoyer, W., Stroucken, T. \& Koch, S. W. Exciton Formation in Semiconductors and the Influence of a Photonic Environment. Phys. Rev. Lett 87, 176401 (2001). 
11. Huber, R., Tauser, F., Brodscheim, A., Bichler, M., Abstreiter, G. \& Leitenstorfer, A. How many-particle interactions develop after ultrafast excitation of an electron-hole plasma. Nature 414, 286-289(2001).

12. Beard, M.C., Turner, G.M. \& Schmuttenmaer, C.A. Transient photoconductivity in GaAs as measured by time-resolved terahertz spectroscopy. Phys. Rev. B62, 15764 15777 (2000).

13. Lövenich, R., Lai, C. W., Hägele, D., Chemla, D. S. \& Schäfer, W. Semiconductor polarization dynamics from the coherent to the incoherent regime: Theory and experiment. Phys. Rev. B 66, 045306 (2002).

14. Nuss, M. C. \& Orenstein, J. in Millimeter and Submillimeter Wave Spectroscopy of Solids (ed G. Grüner) 7-50 (Springer Verlag, Berlin, 1998).

15. Dressel, M. \& Grüner, G. Electrodynamics in Solids 61-62 (Cambridge University Press, 2002).

16. Haug, H. \& Koch, S.W. Quantum Theory of the Optical and Electronic Properties of Semiconductors (World Scientific Publ., Singapore, 1994).

17. Gerlach, B., Wüsthoff, J., Dzero, M. O. \& Smondyrev, M. A. Exciton binding energy in a quantum well. Phys. Rev. B58, 10568-10577 (1998).

18. Tzoar, N. \& Platzman, P.M. High-frequency conductivity of a two-dimensional, two-component electron gas. Phys. Rev. B20, 4189-4193 (1979).

19. Wegener, M. et al. Femtosecond dynamics of excitonic absorption in the infrared $\mathrm{In}_{\mathrm{x}} \mathrm{Ga}_{1-\mathrm{x}} \mathrm{As}$ quantum wells. Phys. Rev. B39, 12794-12801 (1989) and references therein. 20. Selbmann, P. E., Gulia, M., Rossi, F., Molinari, E., \& Lugli, P. Coupled free-carrier and exciton relaxation in optically excited semiconductors. Phys. Rev. B54, 4660-4673 (1996). 
21. Siantidis, K., Axt, V. M. \& Kuhn, T. Dynamics of exciton formation for near band-gap excitations. Phys. Rev. B65, 035303 (2002).

22. Oh, I. K. Exciton formation assisted by LO phonons in quantum wells. Phys. Rev. B62, 2045-2050 (2000) and references therein.

23. Betz, M. et al. Nonlinear optical response of highly energetic excitons in GaAs: Microscopic electrodynamics at semiconductor interfaces. Phys. Rev. B65, 085314 (2002).

24. Siarkos A., Runge, E. \& Zimmermann, R. Center of mass properties of the exciton in quantum wells. Phys. Rev. B61, 10854-10867 (2000).

25. Damen, T. C. et al. Dynamics of exciton formation and relaxation in GaAs quantum wells. Phys. Rev. B42, 7434-7438 (1990).

26. Blom, P.W.M., van Hall, P.J., Smit, C., Cuypers, J.P. \& Wolter, J.H. Selective exciton formation in thin $\mathrm{GaAs}_{/} \mathrm{Al}_{x} \mathrm{Ga}_{1-x} \mathrm{As}$ quantum wells. Phys. Rev. Lett. 71, 3878 3881 (1993).

27. Deveaud, B., Sermage, B. \& Katzer, D. S. Free exciton versus free carrier luminescence in a quantum well. J. Phys. Colloq. C5, 11-14 (1993).

28. Kumar, R., Vengurlekar, A.S., Prabhu, S.S., Shah, J. \& Pfeiffer, L.N. Picosecond time evolution of free electron-hole pairs into excitons in GaAs quantum wells. Phys. Rev. B54, 4891-4897 (1996).

29. Landau, L. \& Zeldovich, J. On the relation between the liquid and gaseous states of metals. Acta Physicochimica, U.S.S.R. XVIII No 2-3, 194-196 (1943).

30. Mott, N. F. Metal Insulator Transitions (Taylor and Francis, London, 1990). 


\section{Acknowledgements}

We thank J. Reno (Sandia Natl. Lab.) for growth of the quantum well samples and S. L. Chuang for bandstructure calculations. Very insightful discussions with M. Kira, S. W. Koch, M. Woerner, T. Timusk, and J. Orenstein are acknowledged. This work was supported by the Office of Basic Energy Sciences of the US Department of Energy, the Deutsche Forschungsgemeinschaft and the Alexander von Humboldt Foundation.

Correspondence and requests for materials should be addressed to R.A.K. (e-mail: RAKaindl@lbl.gov). 
Figure 1 Experimental scheme. a, excitation spectrum of electron-hole pairs with energy $\mathrm{E}$ and center-of-mass momentum $\mathrm{K}$ in a semiconductor. The $1 \mathrm{~s}$ and 2s exciton manifolds lie below the continuum of unbound pairs (grey area). Optical pair excitations (blue arrows) can occur only close to $\mathrm{K} \approx 0$ due to the small photon momentum. Scattering of unbound pairs into exciton states (green arrow) involves large momentum transfer. Transitions between internal exciton states (red arrows) probe excitons at center-of-mass momenta well outside the optically accessible range. $\mathbf{b}$, carriers are photoexcited in the quantum well by a near-infrared $(\lambda \approx 800 \mathrm{~nm}$ ) laser pulse of 1 ps duration. A broadband THz pulse of 500 fs duration $(\lambda \approx 100-300 \mu \mathrm{m})$ arrives with time delay $\Delta$ t relative to the pump, and detects the $\mathrm{THz}$ conductivity and dielectric response of the electrons and holes.

Figure 2 Near-infrared and THz properties of GaAs multiple-quantum-wells. a, near-infrared absorption at the fundamental band edge. With increasing energy, the 1s heavy-hole $(\mathrm{HH})$ exciton absorption line $(\mathrm{E}=1.540 \mathrm{eV}$, width $0.8 \mathrm{meV}$ FWHM), the 2s HH, and the 1s light-hole ( $\mathrm{LH}$ ) absorption lines appear followed by band-to-band transitions. The HH-LH splitting of $\approx 11 \mathrm{meV}$ results from carrier confinement and substrate-induced strain. Photoluminescence spectra (not shown) exhibit a $1 \mathrm{meV}$ line width shifted $<0.5 \mathrm{meV}$ relative to the $\mathrm{HH}$ line. After molecular beam epitaxy, the layers were attached to a MgO wafer, and the substrate removed. b,c, induced $\mathrm{THz}$ conductivity $\Delta \sigma_{1}(\mathrm{red})$ and dielectric function change $\Delta \varepsilon_{1}$ (blue), $\Delta t=5$ ps after resonant excitation of $\approx 1 \times 10^{10} \mathrm{~cm}^{-2}$ $\mathrm{HH}$ excitons at lattice temperature $\mathrm{T}_{\mathrm{L}}=6 \mathrm{~K}$. An aperture of $2 \mathrm{~mm}$ ensures high transmission of the long-wavelength $\mathrm{THz}$ radiation. Black lines: internal exciton response calculated from the dipole moment between the quasi-2D exciton wavefunctions $^{16}$, with the height scaled to the experimental value. d,e, corresponding response for excitation into the continuum at $T_{L}=300 \mathrm{~K}$. The 
black lines show a fit with the Drude model $\sigma(\omega)=n e^{2} d_{Q W^{-1}} m^{-1}(1 / \tau-i \omega)^{-1}$, with $\mathrm{n}=2.3 \times 10^{10} \mathrm{~cm}^{-2}, \mathrm{~d}_{\mathrm{QW}}=14 \mathrm{~nm}, \mathrm{~m}=0.067 \cdot \mathrm{m}_{0}$, and $1 / \tau=2 \pi \cdot 1.15 \mathrm{THz}$.

Figure 3 Temperature dependence of $\mathrm{THz}$ dynamics. a, Dynamics of the induced transient conductivity spectra $\Delta \sigma_{1}(\omega)$ for three different lattice temperatures of $T_{L}$ as indicated, after resonant $\mathrm{HH}$ excitation $\left(n \approx 1 \times 10^{10} \mathrm{~cm}^{-2}\right)$. b, Transient spectra for $T_{L}=60 \mathrm{~K}$. The change of conductivity $\Delta \sigma_{1}$ (left panel) and dielectric function $\Delta \varepsilon_{1}$ (right panel) is shown for several pump-probe time delays. The curves within each panel are equally scaled, but for clarity are shifted in height as indicated by the black line. The red lines $(\Delta t=100 \mathrm{ps})$ are the Drude model fit as in Figs $2 \mathrm{~d}, \mathrm{e}$ but with $\mathrm{n}=1.2 \times 10^{10} \mathrm{~cm}^{-2}$ and $1 / \tau=2 \pi \times 0.5 \mathrm{THz}$

Figure 4 Exciton formation. a, nonresonant excitation with 21 meV excess energy at lattice temperature $\mathrm{T}_{\mathrm{L}}=6 \mathrm{~K}(\mathrm{E}=1.561 \mathrm{eV}$, photoexcited density $\mathrm{n} \approx 10^{10} \mathrm{~cm}^{-2}$ ). The change of conductivity (left panel) and dielectric function (right panel) are shown for several time delays $\Delta$ t between the near-infrared pump and $\mathrm{THz}$ probe pulses. Within each panel the curves are equally scaled, but shifted as indicated by the black line. Red lines $(\Delta t=0 \mathrm{ps})$ : Drude model with $\mathrm{n}=2 \times 10^{10} \mathrm{~cm}^{-2}$ and $1 / \tau=2 \pi \times 0.5 \mathrm{THz}$. b, time-evolution of the induced dielectric function response $\Delta \varepsilon_{1}$ at $\omega=1 \mathrm{THz}(4.1 \mathrm{meV})$. Inset: schematic evolution of $e-h$ gas dominated by unbound quasi-particles, yet with excitonic correlations, into a pure population of excitons. 
FIGURE 1

R.A. Kaindl et al.

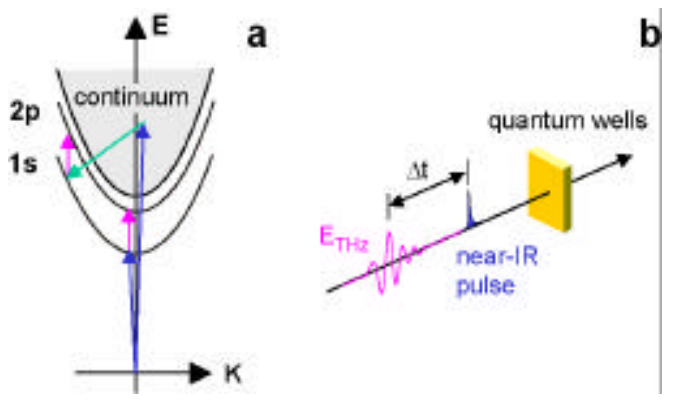




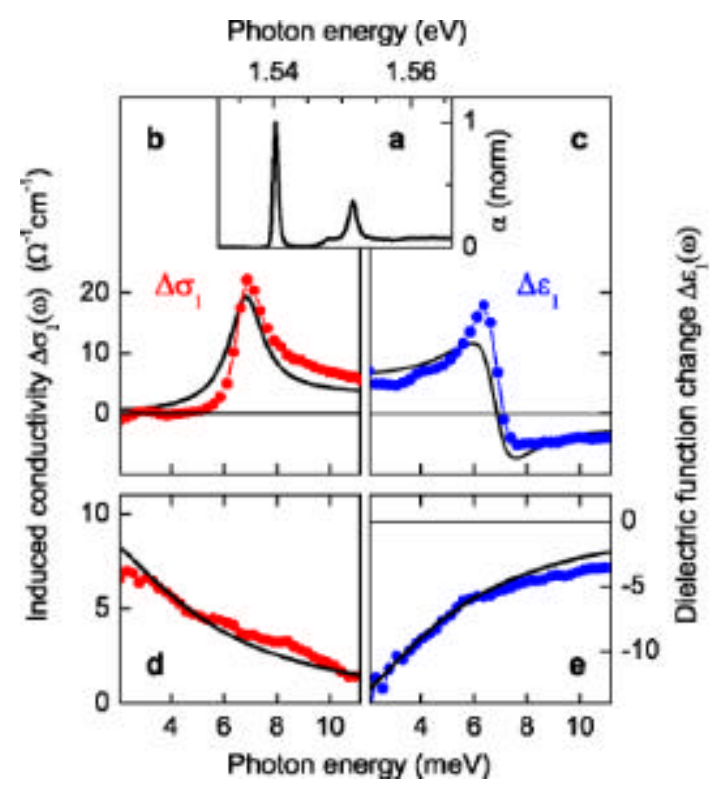


FIGURE 3

R.A. Kaindl et al.
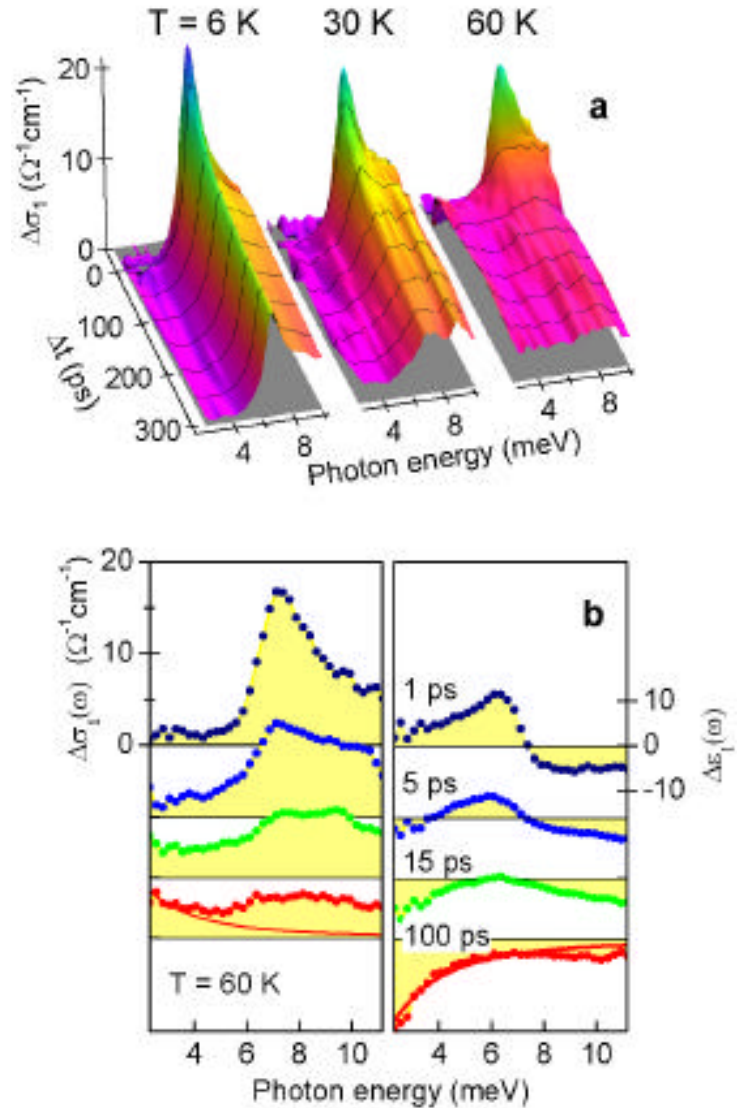

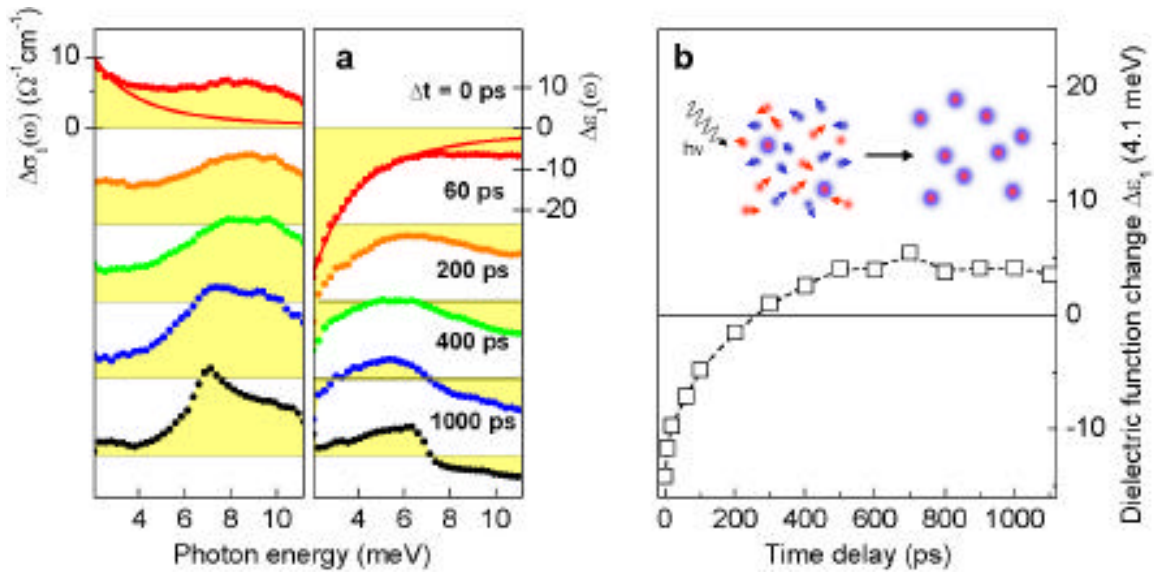